\title{
Numerical model of the nanoindentation test based on the digital material representation of the Ti/TiN multilayers*
}

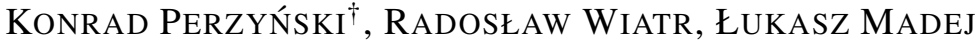 \\ Department of Applied Computer Science and Modeling, AGH University of Science and Technology, 30 Mickiewicza Ave., \\ 30-059 Cracow, Poland
}

\begin{abstract}
The developed numerical model of a local nanoindentation test, based on the digital material representation (DMR) concept, has been presented within the paper. First, an efficient algorithm describing the pulsed laser deposition (PLD) process was proposed to realistically recreate the specific morphology of a nanolayered material in an explicit manner. The nanolayered $\mathrm{Ti} / \mathrm{TiN}$ composite was selected for the investigation. Details of the developed cellular automata model of the PLD process were presented and discussed. Then, the Ti/TiN DMR was incorporated into the finite element software and numerical model of the nanoindentation test was established. Finally, examples of obtained results presenting capabilities of the proposed approach were highlighted.
\end{abstract}

Keywords: pulsed laser deposition; nanolayers; nanoindentation; digital material representation

(C) Wroclaw University of Technology.

\section{Introduction}

In order to improve mechanical, frictional or biocompatibility behavior of well know materials, like titanium (Ti) or titanium nitride (TiN), scientists are trying to develop new manufacturing and processing operations. One of those methods, that provides interesting results, is called the deposition process. During deposition, material is upgraded with new surface layers that are characterized by specific required properties. The main representative of the technology is the Pulsed Laser Deposition (PLD) method, which is a modification of the standard Pulsed Vapour Deposition (PVD) approach. The main idea of the PLD is based on a high-power laser beam that is focused periodically onto the target material provoking instantaneous evaporation and ionization of the surface atoms [1-3]. Products of such ionization consist of atoms, electrons and ions, which are driven away at a high speed from the target plate into the precisely

\footnotetext{
${ }^{*}$ This paper was presented at the $4^{\text {th }}$ National Conference on Nano- and Micromechanics (KKNM) Conference, Wrocław, Poland, 8-10 July, 2014.

†E-mail: kperzyns@agh.edu.pl
}

controlled vacuum. Finally, the particles strike with a high speed into the surface of the substrate material and start to nucleate and grow. Thin films grow with the same chemical composition as the evaporated material. This process gives possibilities for fast and efficient production of layers used in different kinds of engineering materials.

Deposited layers have usually different mechanical properties in comparison to substrate material, what can significantly change the behavior of the structures under exploitation conditions. However, the layers have usually nanometer scale, which causes problems with performing standard plastometric tests that are used to evaluate material properties. One of the possibilities to solve this issue is application of specially designed tests, like microcompression [4] or nanoindentation [5]. Nanoindentation can provide valuable information regarding mechanical and strength behavior of nanostructure composites and is quite widely available in comparison with the mentioned microcompression tests that require very sophisticated equipment.

Strength analysis is extremely important when biomedical applications of mentioned materials 
are considered. The nanoindentation test gives possibilities to receive information about maximal level of deformation, which can be safely applied to the deposited structure without the danger of failure. Additionally, information regarding mechanical parameters, like Young modulus or nanohardness, can be obtained. However, experimental procedure is highly expensive because of specific equipment that has to be used at this length scale. Recently, possibilities provided by the atomic force microscope for precise control of the indentation test gave an opportunity to perform nanoindentation even at the nanoscale $\left(10^{-9} \mathrm{~m}\right)$ (Fig. 1).

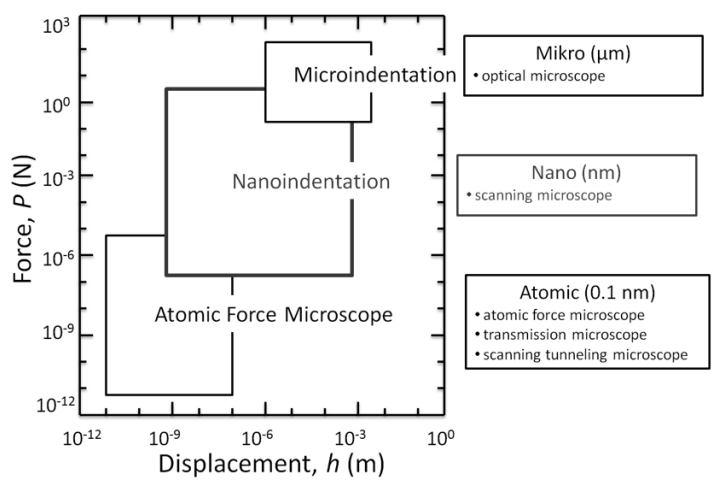

Fig. 1. Range of forces and displacements that can occur in indentation tests.

Nanoindentation during the last fifteen years was also used for investigation of $\mathrm{Ti}$ and $\mathrm{TiN}$ nanolayers. Metallic nanolayers on the basis of titanium alloy (Ti), and ceramics on the basis of titanium nitride (TiN) are often used, for example, in tool industry (protective outer layer of tools), automotive (car body protective layer) or bioengineering (protective implants, prosthesis or bionanolayers). TiN is fully biocompatible, that is why it is often deposited on, e.g., prosthesis or implants.

The main problem with the nanolayered structures is related with selection of appropriate size of such layers. Well-designed size of nanolayers will protect important parts of the components and will prevent from continuity lose, which can be dangerous for, e.g., patients.

Recent scanning microscope analysis of nanolayers revealed that microstructure of deposited layers obtained with the use of, e.g., PLD technique has a well developed columnar structure and clearly visible surface inhomogeneities between the substrate and subsequent nanolayers [6]. In the works [7-13], authors performed nanoindentation tests of the TiN and Ti nanolayers to analyse behavior of the layers microstructure. Furthermore, TEM observations of deformed structures showed, e.g., that damages propagate along the borders between the columns (Fig. 2). Such behavior dramatically changes material resistance at larger deformation degrees.

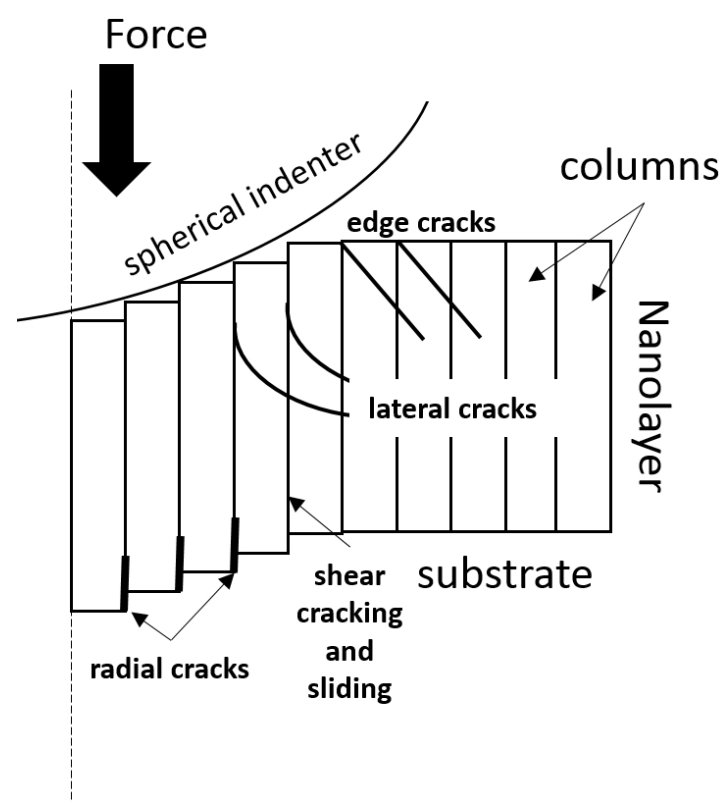

Fig. 2. Damage evolution observed in the nanolayers during nanoindentation test.

Unfortunately, experimental analyses at these length scales are usually very expensive. That is why authors decided to develop a numerical model of the nanoindentation test to investigate material behavior under loading conditions that would be able to support experimental research. However, commonly used numerical models neglect the inner structure of deposited layers [14-17] what depreciates the quality of obtained data. Deposited material is usually defined as isotropic without taking into account columns and surface wrinkling. Thus, simplified models cannot give sufficient information about material resistance to deformation. Thus, development of the model, which precisely 
maps the nanolayer morphology and inner structure seems extremely relevant and important because of the need for obtaining meaningful results comparable with studies made on the real material.

In the literature $[18,19]$ a number of articles can be found, where authors simulate the deposition process with the Monte Carlo methods. However, these microstructures have not been used during subsequent modeling of deformation with the finite element method. That is why, in the present work, authors decided to develop a numerical model of the nanoindentation test that includes a detailed virtual representation of the layers morphology. For that reason the cellular automata (CA) model of the deposition process was developed, which provided a digital representation of the layers for subsequent numerical modeling as a function of deposition process parameters. As a result, the complex nanoindentation model provides basic knowledge about material response due to local deformation conditions.

\section{Cellular automata model of the PLD deposition process.}

The main idea of the cellular automata technique is to divide a specific part of material into one-, two-, or three-dimensional lattice of finite cells. Each cell in this space is called a cellular automaton, while the lattice of the cells is known as a cellular automata space. Each cell in the CA space is additionally surrounded by a set of neighbours, which affect one another. The cells interactions within the CA space are based on the knowledge defined, while studying a particular phenomenon. In every time step, the state of each cell in the lattice is determined by the previous states of its neighbours and the cell itself by a set of precisely defined transition rules $f$ :

$$
Y_{i}^{t+1}=f\left(Y_{j}^{t}\right)
$$

where: $j \in N(i)$ - surroundings of the $i^{\text {th }}$ cell, $Y_{i}-$ state of the $\mathrm{i}^{\text {th }}$ cell.

Since the transition rules control the cells behavior during calculations, the proper definition of these rules in the process of designing a CA model affects the accuracy of this approach.

The implemented CA model of the deposition process is divided into three separate steps: formation of new molecules, deposition of the molecules and surface diffusion of molecules after deposition at the substrate.

The model is implemented in the 2D space, where molecules positions are described by (xy) coordinates. Each CA cell represents a single molecule and contains information on cell state, ID layer (assigns cell to the separate layer), ID material (assigns cell to a specific material), ID island (assigns cell to a particular growing island) and actual energy value $E_{x y}$. The model operates on two kinds of neighbourhoods: Moore and von Neumann. Evolution of the CA space is realized by the defined transition rules, which allow one to transform the physics of the deposition process into a series of probabilistic transition rules. The present model is simplified and behavior of the molecules at the surface is limited only to deposition (adsorption) and migration along the surface (surface diffusion).

There are five states describing each particular CA cell: Empty, Surface, Molecule, Molecule_ $i$ and Freezed, as seen in Fig. 3.

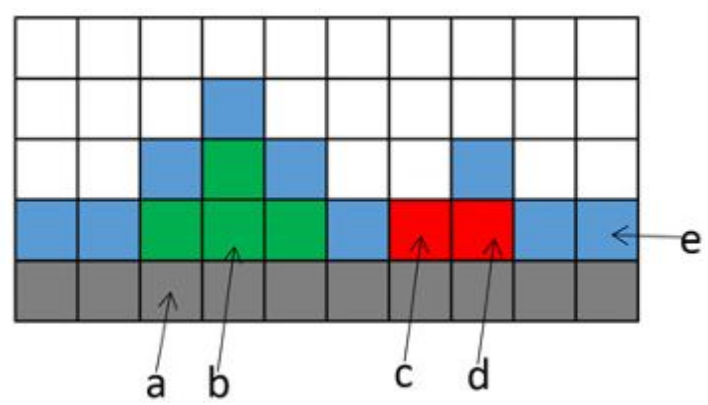

Fig. 3. Possibilities of the CA states (von Neumann neighbourhood), a) Surface b) and d) Freezed, c) Molecule and e) Molecule_i.

The state Surface is a fixed state that represents a substrate material, thus the cell cannot move from this state to the other. The Molecule state describes molecules that have already been deposited at the surface and they are carrying information about the material: ID of the layer and ID of the island to 
which they belong to, e.g., cell $b$ from Fig. 3 belongs to different island than cells $\mathrm{c}$ and $\mathrm{d}$. It is also assumed that only cells in the state Molecule can change position along the surface, e.g., cell $\mathrm{c}$ can migrate, while cells $\mathrm{b}$ and $\mathrm{d}$ are in Freezed state (all neighbours occupied by other cells). Additionally, an intermediate Molecule state was defined - Molecule_ $i$, that represents a new unadsorbed molecule in a Molecule state. These cells do not yet belong to any island and they can diffuse along the surface and can be adsorbed by existing islands. However, if in the neighbourhood of such cell there are no cells in the Molecule or Freezed state, then this cell can also be a precursor of a new island growth. As mentioned, the developed model is based on three major steps (Fig. 4).

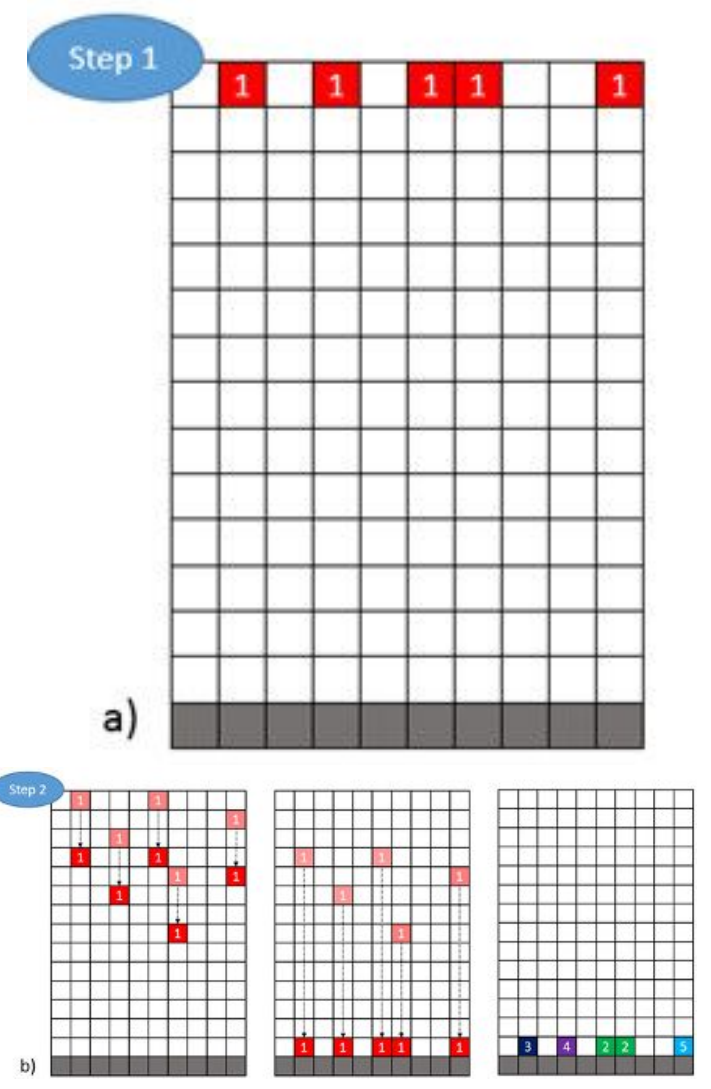

Fig. 4. Three major algorithm steps: (a) creation of new molecules, (b) deposition at the substrate (c) surface diffusion.

The first step replicates the formation of new molecules during the incidence of the laser beam on the target, which is a source of molecules deposited at the surface. The model assumes complete absence of the phenomena occurring prior to deposition of particles at the surface. The process is based only on the parameter $\theta$, defining the density of particles reaching the surface in a given time step. The value depends on the laser parameters, such as power and pulse length [20]:

$$
\theta(t)=\left(\frac{F_{i}}{f}\right) e^{-\frac{t}{\Gamma}}
$$

where: $\mathrm{t}$ - laser impulse time, $\mathrm{F}_{\mathrm{i}}$ - average power of laser, $f-$ frequency of the laser, $\Gamma$ - coefficient of inertia.

Factor $\Gamma$ is determined depending on the frequency and duration of the laser pulse. For frequencies $\sim 50 \mathrm{~Hz}$ and a pulse length of $\sim 10 \mathrm{~ns}$ value $\Gamma$ will be 0.01 [21]. As previously mentioned, despite the complexity of transportation phase, molecules are deposited on the surface in fairly uniform manner. New molecules are deposited in the CA space in the first empty available location in consecutive rows, as seen in Fig. 4a. The initial x coordinate of the new molecule is selected randomly. Number of the new molecules generated during a single time step is calculated as:

$$
X Y \theta(t)=N_{m}
$$

where: XY - width and height of the working area, $\theta(t)$ - density of the molecules generated at the time $\mathrm{t}, \mathrm{N}_{\mathrm{m}}$ - number of molecules.

Each new cell representing a molecule is additionally described by a set of internal variables: ID attributed to the material (ID material), ID attributed to the layer (ID layer) and ID attributed to the island (ID island). When a molecule is not connected to another cell (not yet belongs to any of the islands) it receives ID island equal to 1 .

The second step is used to describe the process of deposition: falling of the molecules and their adsorption at the surface. The falling process is modelled in a simplified manner, where in each time step the molecule can move by a distance of a single CA cell down to the surface, where a CA cell changes the state to Molecule. After deposition, each cell in the Molecule state examines its 
neighbourhood and if there is no other cell in the Molecule state it will join to represent the same island, if not, the molecule will initiate a separate island as seen in Fig. 4b.

The third step of the algorithm is related to molecules deposited at the surface that are in the Molecule state. These cells can change the position along the surface in each time step. New position is evaluated within the limited area defined by the diffusion rate $\mathrm{R}$ (Fig. 5):

$$
R=\frac{M D f}{E_{x y}} \Delta T
$$

where: MDf - material constant that represents molecule diffusivity in the range $\langle 0, \infty\rangle, \Delta \mathrm{t}$ - time increment, $E_{x y}$ - molecule energy in a particular location:

$$
E_{x y}=\sum_{i=0}^{n_{x y}}(r+1-\Delta d)
$$

where: $\mathrm{r}$ - neighbour radius, $\Delta \mathrm{d}$ - distance between neighbours, $\mathrm{n}_{\mathrm{xy}}-$ number of neighbours.

The movement of molecules is driven by reaching the lowest energy value (Fig. 6) in the investigated area. The implemented nondeterministic algorithm reflects the chaotic way that molecules diffuse during the process.

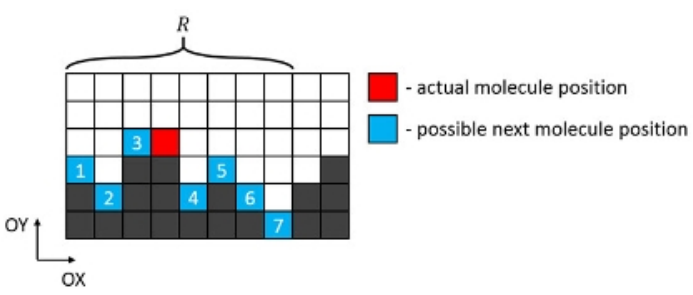

Fig. 5. Schematic illustration representing local minimum algorithm.

To determine parameters of the proposed model for the Ti and TiN materials, authors generated a series of nanolayers and compared them with experimental data [22]. Identified model parameters are gathered in Table 1.

Application of the developed algorithm to generation of digital material representation (DMR) model of Ti/TiN layers is presented in the following chapter.

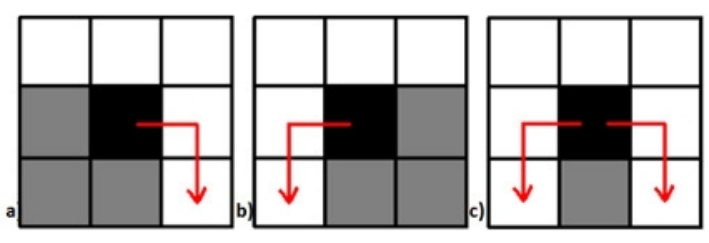

Fig. 6. Schematic possibilities for surface diffusion with using local energetic minimum algorithm.

Table 1. Diffusion parameters for Ti and TiN materials.

\begin{tabular}{cccc}
\hline \multicolumn{2}{l}{ Number of layer } & Material & Deposition time [min] \\
\hline \hline 1 & $\mathrm{Ti}$ & 20 & 50 \\
2 & $\mathrm{TiN}$ & 20 & 20 \\
\hline
\end{tabular}

\section{Digital material representation of two layers $\mathrm{Ti} / \mathrm{TiN}$ structure.}

Digital material representation (DMR) is a material description based on measurable quantities that provides the necessary link between simulation and experiment [23]. The DMR is expected to create a possibility of analysis material behavior in the conditions which are difficult or even impossible to be monitored experimentally at the present state of equipment. The main objective of the DMR is creation of a digital representation of microstructure with its features represented explicitly to match a real microstructure morphology. Thus, based on the previously presented algorithm for deposition of nanolayer coatings, a DMR model of a two-layer structure consisting of Ti/TiN material was created with the parameters presented in Table 2.

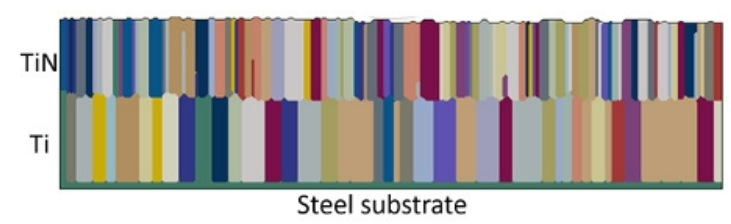

Fig. 7. Digital material representation of Steel/Ti/TiN nanostructure.

After deposition process on a steel substrate, a DMR consisting of $60 \mathrm{~nm}$ in height nanolayers of Ti and TiN was obtained as seen in Fig. 7. 
Table 2. Parameters used for deposition Ti and TiN nanolayers.

\begin{tabular}{cccccc}
\hline $\begin{array}{c}\text { Number } \\
\text { of layer }\end{array}$ & Material & $\begin{array}{c}\text { Deposition } \\
\text { time [s] }\end{array}$ & $\begin{array}{c}\text { Laser } \\
\text { power [J] frequency [Hz] }\end{array}$ & $\begin{array}{c}\text { Laser } \\
\text { the target [ns] }\end{array}$ \\
\hline \hline 1 & $\mathrm{Ti}$ & 240 & 0.6 & 50 & 10 \\
2 & $\mathrm{TiN}$ & 240 & 0.6 & 50 & 10 \\
\hline
\end{tabular}

The possibility to assign material properties to a particular Ti or TiN column is the main advantage of the developed DMR model. Due to the fact that only cold deformation processes were investigated in the present work, authors decided to use a simple isotropic elasto-plastic nonlinear hardening rule:

$$
\begin{gathered}
\sigma_{i}=E \varepsilon_{i} \text { for } 0<\sigma_{i}<\sigma_{Y} \\
\sigma_{i}=C \varepsilon_{i}^{n} \text { for } \quad \sigma_{i}>\sigma_{Y}
\end{gathered}
$$

where: $\mathrm{E}-$ Young modulus, $\sigma_{\mathrm{i}}$ - equivalent stress, $\sigma_{\mathrm{Y}}-$ yield stress, $\varepsilon_{\mathrm{i}}-$ equivalent strain, $\mathrm{C}, \mathrm{n}-$ constants.

In this approach, the size of the yield space is fixed in the stress space, while only radius expands in subsequent steps. In the DMR model there is a possibility to capture differences in the grain flow due to various crystallographic orientations of columns. It was done through diversification in the flow curves for each particular grain. The diversification was introduced using the random Gauss distribution (Fig. 8). To obtain such a diversification the authors specified the range in which the flow stress parameters can change, and the obtained values were then automatically assigned to subsequent grains. The Gaussian distribution was used to generate material properties for all Ti and TiN columns existing in the microstructure. Mechanical properties for diversification were selected based on [24].

The generated morphology was subjected to a discretization algorithm, creating a finite element mesh as seen in Fig. 9. The non-uniform mesh was created using a DMRmesh software developed by the authors in [25]. The FE mesh was highly refined along the columns boundaries to properly capture significant solution gradients that were expected in these regions due to large differences in hardening behaviour of Ti and TiN layers.

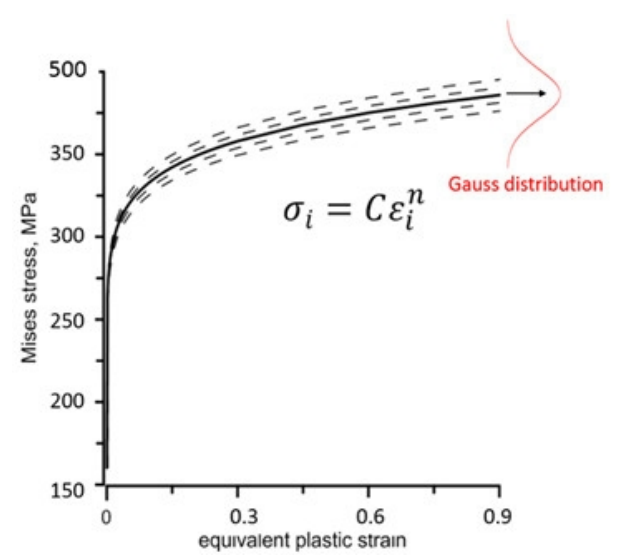

Fig. 8. Schematic representation of curves generated with the Gaussian distribution function for $\mathrm{Ti}$ columns.

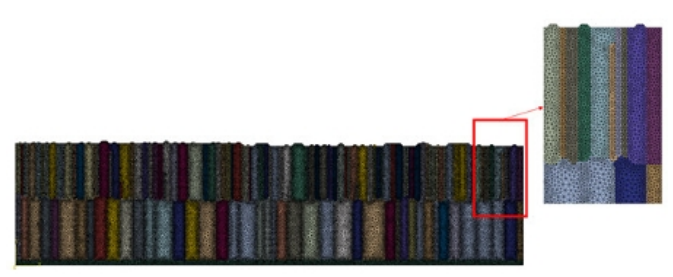

Fig. 9. Finite element discretization made on the Ti/TiN nanolayers.

\section{Numerical nanoindentation test}

Numerical model based on the experimental procedure of the nanoindentation test was developed in the commercial ABAQUS package. The developed model is based on the generated above DMR. During the test, a spherical nanoindenter with $100 \mathrm{~nm}$ tip radius was used. Illustration of the developed model is shown in Fig. 10. To maintain continuity of the presented structure, symmetric boundary conditions were applied. Additionally, displacement of the steel substrate was fixed in every direction, while the indenter was displaced into nanolayers up to the level of $30 \mathrm{~nm}$. 


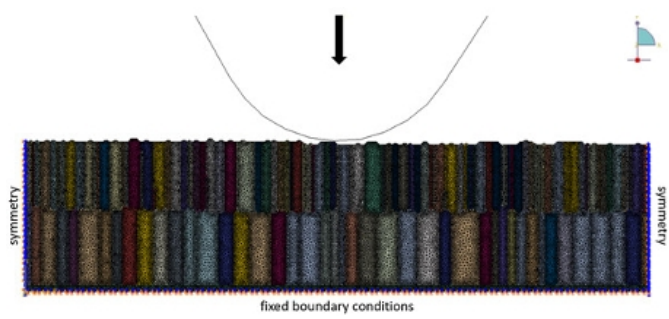

Fig. 10. Assembly with used boundary conditions.

Inhomogeneous distribution of strain accumulation in various columns is clearly visible in Fig. 11. In the TiN layer, especially between columns under the nanoindenter working area, large strain and stress irregularities can be observed. Those stress and strain concentration zones can easily change into failure initiation zones and lead to destruction of the nanolayered material.

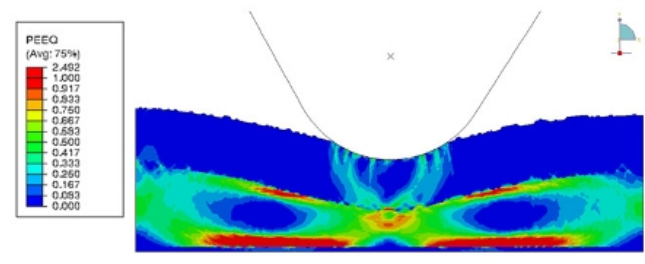

(a)

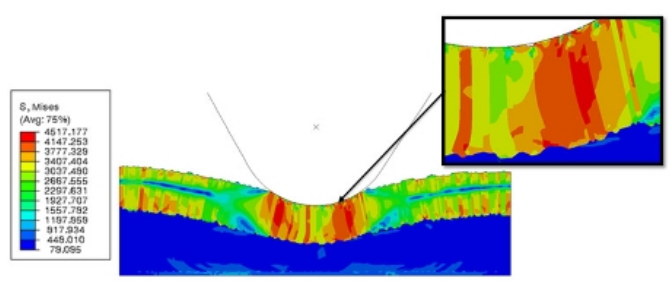

(b)

Fig. 11. Strain and stress distribution after nanoindentation test.

Thus, these results clearly highlight the importance of taking into account the reliable representation of layers morphology.

\section{Conclusions}

Based on the presented results it can be concluded that:

- cellular automata is an effective method for modeling deposition process and can provide representation of columnar morphology of deposited layers,

- despite simplifications introduced into the CA model, obtained results replicate complex morphology of deposited layers,

- development of the nanoindentation numerical model based on DMR concept provides a possibility to predict local stress/strain inhomogeneities that are crucial when material failure is investigated

- plans for the future works are mainly focused on extension of the proposed deposition model into the 3D space.

\section{Acknowledgements}

Work within 2015 Statute Research No. 11.11.110.291 at the Department of Applied Computer Science and Modeling at AGH. The calculations were performed at the ACK Cyfronet: MNiSW/IBM_BC_HS21/AGH/076/2010.

\section{References}

[1] Major B., Ablacje i osadzanie laserem impulsowym, Akapit, Kraków, 2002.

[2] Sarna J., Kustosz R., Major R., LACKner J.M., Major B., B. Pol. Acad. Sci.-Tech., 58 (2010), 329.

[3] MAJOR B., Materials science in heart disease treatment, Nanostructural materials for implants and cardiovascular biomedical devices, CARDIOBIOMAT, 2012.

[4] Madej L., Wang J., Perzynski K., Hodgson P.D., Comp. Mater. Sci., 95 (2014), 651.

[5] Perzynski K., Madej L., Wang J., Kuziak R., Hodgson P.D., Metall. Mater. Trans. A, 45 (2014), 5852.

[6] Thornton J.A., J. Vac. Sci. Technol., 11 (1973), 666.

[7] Ma K. J., Bloyce A., Bell T., School Metall. Mater, 76 - 77 (1995), 297.

[8] Varst Van Der P.G.T., With DE G., Thin Solid Films, 384 (2001), 85.

[9] Bhowmick S., Kale A.N., Jayaram V., Biswas S.K., Thin Solid Films, 436 (2003), 250.

[10] Ma L., Cairney J., McGrouther D., Hoffman M., Munroe P., Thin Solid Films, 515 (2007), 3190.

[11] Tilbrook M.T., Paton D.J., Xie Z., Hoffman M., Acta Mater., 55 (2007), 2489.

[12] Lackner J., Waldhauser W. Alamanou A., TeICHERT C., Schmied F., MAJor L., MAJor B., $B$. Pol. Acad. Sci.-Tech., 58 (2010), 281.

[13] Major L., Arch. Civ. Mech. Eng., 14 (2014), 615.

[14] Lichinchi M., Lenardi C., Haupt J., Vitali R., Thin Solid Films, 312 (1998), 240.

[15] Huang X., Pelegri A.A., Compos. Sci. Technol., 67 (2007), 1311. 
[16] Pelegri A.A., Huang X., Compos. Sci. Technol., 68 (2008), 147.

[17] Kopernik M., Milenin A., Major R., LAckner J.M., Mater. Sci. Tech.-Lond., 27 (2011), 604.

[18] Zhang Q., Zhu J., Tan J., Yu G., Wu J., Zhu J., XIAO D., Vacuum, 81 (2006), 539.

[19] Tan X., Zhou Y. C., Zheng X. J., Surf. Sci., 588 (2005), 175.

[20] Warrender J. M., AzIz M. J., Appl. Phys. A-Mater., 79 (2004), 713.

[21] SHIN B., Study of Thin Film Growth Kinetics of Homoepitaxy by Molecular Beam Epitaxy and Pulsed Laser Deposition, Harvard University Cambridge, Cambridge, 2007.
[22] Major L., Morgiel J., Major B., Lackner J.M., Surf. Coat. Tech., 200 (2006), 6190.

[23] Szyndler J., Madej Ł., Comp. Mater. Sci., 96 (2015), 200.

[24] Perzynski K., Major Ł., Kopernik M., Madej Ł., PIETRzYK M., Inżynieria Materiałowa, 31 (2010), 735.

[25] Kruzel F., Madej L., Perzynski K., Banas K., Int. J. Multiscale Com., 12 (2014), 257.

Received 2014-10-22

Accepted 2015-02-24 